\title{
SITUACIÓN LABORAL DE LOS JÓVENES EN EL GRAN RESISTENCIA Y CORRIENTES, ARGENTINA, EN EL PERÍODO 2010-2013
}

\author{
PABLO BARBETTI \\ JOSÉ ANTONIO POZZER \\ BLANCA SOBOL
}

UNIVERSIDAD NACIONAL DEL NORDESTE

ARGENTINA.

"Tengo un sueño, un solo sueño, seguir soñando. Soñar con la libertad, soñar con

la justicia, soñar con la igualdad y ojalá ya no tuviera necesidad de soñarlas". Martin Luther King, Jr. 


\title{
Instituto de Investigaciones Educativas (Facultad de Humanidades) y Departamento de Ciencias Sociales (Facultad de Ciencias Económicas) de la Universidad Nacional del Nordeste
}

Este trabajo se desarrolla en el marco del proyecto 12Hoo7: "Trayectorias socio-educativas y laborales de jóvenes de sectores vulnerables de Chaco y Corrientes”, acreditado por la Secretaría General de Ciencia y Técnica de la Universidad Nacional del Nordeste.

\section{RESUMEN}

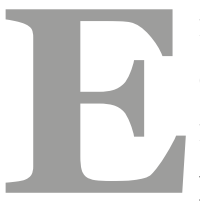

n este artículo se presenta un estudio descriptivo del comportamiento del mercado laboral de los jóvenes en los aglomerados urbanos Gran Resistencia y Corrientes durante el período 2010 - 2013. Se basa en el análisis de algunas de las principales variables relacionadas con la problemática socioeducativa y laboral de los jóvenes, para lo cual se ha tomado como fuente de datos secundarios la Encuesta Permanente de Hogares del INDEC correspondientes a los $4^{\circ}$ trimestres de los años mencionados.

Palabras clave: juventudes, mercado de trabajo, inserción laboral.

\begin{abstract}
This paper presents a descriptive study of labor market behavior of young people in urban areas Corrientes and Resistencia occurs during the period 2010- 2013. It is based on the analysis of some key variables related to social, educational and employment problems of young people, which is taken as a source of secondary data from the Permanent Household Survey INDEC corresponding to the 4 th quarters of those years.
\end{abstract}

Keywords: youth, labor market, job placement.

\section{INTRODUCCION}

En este artículo nos proponemos describir el mercado laboral de los jóvenes de 18 a 24 años en los aglomerados urbanos Gran Resistencia y Corrientes durante el período 2010-2013. 
Los jóvenes constituyen un grupo que desde hace décadas viene enfrentando dificultades a la hora de conseguir un empleo, y sobre todo un empleo de calidad. Si bien esta situación se da a nivel mundial, interesa describir su situación en la etapa posterior a la crisis financiera del 2008/2009, la cual significó una retracción de la economía con su consecuente repercusión sobre el mercado de trabajo (Monteforte y Jaccoud, 2013), y que además coincide con un momento en que en Argentina, y en particular en los aglomerados mencionados, se implementan algunas políticas de empleo y de formación profesional dirigidas a este grupo (Vezza y Bertranou, 2011; Barbetti et. al., 2014).

Partiendo del supuesto que las condiciones estructurales afectan los marcos de oportunidades de los sujetos y que por lo tanto inciden en sus distintos procesos vitales ${ }^{1}$, distintos antecedentes señalan la complejización del proceso de transición a la vida adulta. Durante décadas, este pasaje, enmarcado en las instituciones educativas, familiares y laborales no presentó mayores dificultades, sin embargo las transformaciones más recientes en el mercado de trabajo operaron en la dilatación y precarización del proceso (Jacinto, 2010). Esto implicó que las trayectorias ocupacionales de los jóvenes se vieran más afectadas por la incertidumbre y por la circulación entre posiciones laborales inestables y desprotegidas y en particular, el NEA, constituye una de las regiones que presentan peores indicadores respecto a la precariedad laboral juvenil (Golovanevsky, 2013).

En este marco, el análisis se centra en la revisión de algunas de las principales variables relacionadas con la problemática socioeducativa y laboral, para lo cual se ha tomado como fuente las bases de microdatos individuales de la Encuesta Permanente de Hogares (EPH, INDEC) correspondientes al $4^{\circ}$ trimestre de los años 2010 y 2013 y se organiza en cuatro secciones: en la primera realizamos una introducción a la problemática que dio origen al estudio, incluyendo la revisión de algunos antecedentes. En la segunda presentamos una descripción de algunos indicadores generales de la población económicamente activa de la Región NEA, mientras que en la tercera avanzamos en la caracterización específica del mercado laboral juvenil de los aglomerados elegidos. Finalmente, resumimos los principales hallazgos expuestos en el trabajo.

\section{ALGUNAS CONSIDERACIONES GENERALES SOBRE LA SITUACIÓN ACTUAL DEL EMPLEO JUVENIL.}

Como es sabido, el acceso de los jóvenes al mundo del trabajo y la permanencia en el mismo constituye, en las últimas tres décadas, una problemática que se manifiesta a nivel global y que ha sido objeto de numerosos estudios regionales e internacionales.

\footnotetext{
${ }^{1}$ Casal (1996) señala que la transición de los jóvenes a la vida activa se analiza mediante las trayectorias vitales a partir de elecciones y decisiones racionales, pero bajo determinaciones del entorno próximo, estructurales del contexto amplio, y otras de orden cultural y simbólico.
} 
A pesar de los diversos tipos de intervenciones que se han realizado a lo largo de todo este tiempo, este colectivo es el que recibe el mayor impacto de las consecuencias derivadas de las crisis macroeconómicas ${ }^{2}$. En esta línea, un informe reciente de la OIT (2013a) muestra que el debilitamiento de la recuperación económica mundial en los años 2012 y 2013 agravó la situación del empleo juvenil, dificultándose aún más el acceso al empleo para jóvenes que buscan trabajo, a tal punto que muchos renuncian a seguir buscando o bien a ser menos selectivos con los empleos que están dispuestos a aceptar (accediendo en consecuencia a trabajos a tiempo parcial o de menor calidad en cuanto a sus condiciones de trabajo). En este contexto, la tasa mundial de desempleo juvenil, que había disminuido del 12,7\% en 2009 al 12,3\% en 2011, aumentó al 12,6\% en 2013. Por su parte, para 2018, se prevé una tasa mundial de desempleo juvenil de un 12,8\%, así como un aumento de las diferencias de una región a otra (OIT a, op. cit).

Un diagnóstico bastante similar es el que refleja la literatura que analiza esta problemática en el caso específico de América Latina. La misma indica que, a pesar de los avances económicos y políticos, que se dieron en gran parte de los países desde inicios del siglo XXI y que plantean condiciones sociales favorables para un mayor desarrollo de este colectivo ${ }^{3}$, no se produjo una mejoría sustantiva en la situación laboral de los jóvenes. En algunos países se evidencia una mejora relativa en los indicadores laborales para este grupo pero persiste o se incrementa la precaria inserción laboral (OIT, 2013b; Vezza y Bertranou, op. cit.). A su vez, las investigaciones también plantean que los orígenes y causas del problema son múltiples e incluyen explicaciones vinculadas tanto con la oferta como con la demanda (Weller, 2006, Pérez, $2007^{4}$ ), no obstante todos coinciden en el hecho de que quienes se encuentran especialmente afectados son aquellos en mayor situación de vulnerabilidad social.

Para el caso específico de la Argentina, Salvia (2013) sostiene que el actual escenario crítico ha estado precedido por un importante crecimiento económico y del consumo interno, lo cual generó una mejora en materia de empleo y de calidad de vida a nivel general de la población.

Sin embargo, en este escenario existen dos etapas marcadas por la evolución del mercado de trabajo, de 2003 a 2008, y de 2009/10 a la actualidad. La primera etapa se enmarca en el

\footnotetext{
${ }^{2}$ En casi la totalidad de los informes consultados se señala, como una constante, que los jóvenes tienen el doble y, en ocasiones, casi el triple de probabilidades que los adultos de estar desempleados. A escala mundial, la relación entre la tasa de desempleo de los jóvenes y la de los adultos, de un 2,7 por ciento en 2013, apenas ha registrado variaciones en los últimos años.

${ }^{3}$ Con más años de educación que las generaciones previas, más manejo de las nuevas tecnologías y más adaptables a las cambiantes condiciones de las sociedades en comparación con los adultos, este grupo etario tendría mayores oportunidades de acceder a mejores condiciones laborales y de incorporarse a trayectorias laborales ascendentes (OIT, Panorama Laboral 2013)

${ }^{4}$ Entre los diversos factores que inciden en el acceso a un puesto de trabajo, Pérez (op. cit.), señala los siguientes: el nivel y las fluctuaciones de la demanda global, la importancia del empleo en el crecimiento económico, la existencia de instituciones y legislación favorable, la extensión y calidad de la educación y de la formación profesional, la experiencia laboral previa, las prácticas de reclutamiento y selección por parte de las empresas, el origen social de los jóvenes y variables individuales (edad, sexo, expectativas, intereses, etc.). Por lo mismo Weller (op. cit) plantea que no existe un problema de inserción laboral común para todos los jóvenes, sino una variedad de problemas específicos y que los retos que enfrentan jóvenes de distintos género, nivel educativo, trasfondo socioeconómico, cultural, étnico, difieren marcadamente.
} 
efecto del cambio en los precios relativos producido por la devaluación y la reactivación de algunas actividades industriales, verificándose tasas exponenciales de crecimiento del producto, el empleo y una importante recuperación del salario real ${ }^{5}$ (Monteforte y Jaccoud, op. cit.). A esto se sumó una ampliación de las reformas educativas, de los sistemas de formación profesional y de los programas activos de capacitación y empleo dirigidos a los jóvenes a cargo del Estado.

La segunda etapa (post crisis económica mundial 2008/9) ${ }^{6}$ dista de la primera en tanto muestra signos de estancamiento en las principales variables tanto macroeconómicas como del mercado de trabajo, reflejado en el escaso crecimiento del empleo, lo cual abre nuevos espacios de incertidumbre. Así, el 19 \% de los jóvenes están desocupados, el 20 \% se encuentran subocupados (empleos de indigencia), y sólo un $35 \%$ de aquellos que participan de la población económicamente activa logran un empleo pleno en condiciones económicas y legales de inclusión social (Salvia, op. cit).

Ante la problemática laboral de los jóvenes, en las últimas décadas, las medidas implementadas desde el Estado partieron del diagnóstico parcial y limitado -dominante en otros partes del mundo también-, de que el desempleo juvenil sería sólo la consecuencia de una baja capacidad de empleabilidad ${ }^{7}$ de los jóvenes por su falta de educación adecuada a las necesidades del mercado y de experiencia profesional. No obstante, Jacinto (op. cit.) reconoce que en los últimos años, con las nuevas orientaciones de los Gobiernos, la perspectiva empieza a cambiar hacia una mirada que entiende el desempleo juvenil como un problema estructural. Las políticas, entonces, se proponen objetivos redistributivos y de ampliación de los derechos sociales, intentando superar, al menos a nivel discursivo, visiones estigmatizantes y responsabilizantes de la propia pobreza. Así, en el período considerado por este trabajo, se han identificado la implementación de una serie de programas dirigidos a jóvenes que buscan mediar en la reinserción al sistema educativo y/o la inserción al mercado de trabajo, y se dirigen a jóvenes de edades que van desde los 16-18 años hasta 24-29 años. Así, encontramos ofertas que tienen como objeto el regreso al sistema educativo formal y la finalización del período de escolaridad

\footnotetext{
${ }^{5}$ La etapa de crecimiento sostenido registrada entre 2003 y 2008, en que el Producto Bruto Interno (PBI) aumentó a una tasa de $8,5 \%$ anual en promedio (7,2\% en el caso del PBI per cápita). La recuperación de la economía en general, y de la industria manufacturera en particular, trajo aparejada una importante mejora en la productividad, que aumentó a una tasa promedio anual de $4,1 \%$ entre los años 2003 y 2008; incremento que no se produjo como resultado de la sustitución de capital por trabajo sino en el marco de una significativa expansión del empleo.

${ }^{6}$ Varios analistas (Abeles, 2009; Rial, 2009) señalan que la crisis del año 2008 y 2009 ha socavado los niveles de empleo en la mayoría de los países, y que si bien fue caracterizada como una "crisis financiera", es sin duda una crisis mundial que se extiende a toda la "economía real" de los países y, por ende, a los mercados de trabajo. Aunque para el caso argentino, puso de manifiesto que un shock externo de magnitud no generó una crisis de balanza de pagos ni al sistema financiero local (Abeles, op.cit.), lo cual se explicaría en la no participación de los mercados financieros internacionales.

${ }^{7}$ Vinculada a la inadecuación entre los contenidos educativos aprendidos, las capacidades laborales ofertadas y las nuevas demandas tecnológico-organizacionales que generan las empresas; a lo que se suman las trabas que fijan aquellos mercados de trabajo regulados por instituciones laborales muy rígidas, las cuales ponen límites a la libertad contractual pero también a los procesos de entrenamiento, experiencia y formación laboral de los jóvenes en el campo del trabajo.
} 
obligatoria (primaria y secundaria), y que en algunos casos también incluyen formación para el trabajo en propuestas tales como el Plan Fines y Programa Educación Media y Formación para el Trabajo (2009), ambos del Ministerio de Educación. Por su parte, la oferta del Ministerio de Trabajo, Empleo y Seguridad Social del Programa Jóvenes con Más y Mejor Trabajo (2008), propone una serie de componentes que incluyen terminalidad educativa, formación profesional, pasantías e intermediación laboral (Barbetti y otros, op. Cit.).

Conjuntamente, se identifican otras políticas que si bien pueden no estar dirigidos exclusivamente a jóvenes o a la finalización de ciclos básicos, favorecen su permanencia o el retorno al sistema educativo, tales como la Asignación Universal por Hijo (2009) y el recientemente implementado Programa de Respaldo a Estudiantes de Argentina PROG.R.ES.AR (2014).

Ahora bien, retomando la revisión del comportamiento del mercado laboral juvenil, Golovanevsky (op. cit.), por su parte, si bien coincide con el diagnóstico respecto de los indicadores del mercado de trabajo en Argentina, señala que la evolución de las distintas regiones y de los distintos grupos etarios no ha sido equivalente en todos los casos. En particular las Regiones Noroeste (NOA) y Nordeste (NEA) muestran los índices más preocupantes de precariedad del trabajo juvenil.

Específicamente al caracterizar la región NEA, varios analistas (Panaia y San Martín, 2004; Kiss, 2005; Sobol, 2007) señalan ciertas particularidades socio históricas ${ }^{8}$ que contribuyen al acrecentamiento de los procesos de vulnerabilidad del mercado de trabajo, ubicando a la misma como un área de escaso desarrollo y con alto grado de informalidad y precariedad en las ocupaciones, condiciones de las cuales los jóvenes no están exentos.

Es en este marco, surge el interrogante respecto al comportamiento reciente del mercado laboral de los jóvenes en los aglomerados urbanos Gran Resistencia y Corrientes (período 2010 - 2013).

Cabe señalar que para este trabajo se decidió considerar como categoría etaria dentro de la juventud a la población de jóvenes comprendida entre 18 a 24 años de edad 9 , quienes estarían atravesando el momento de transición del sistema educativo al mercado de trabajo. Además, este grupo etario es la población objetivo de varias políticas de empleo y formación para el trabajo identificadas en estudios previos (Barbetti et. al., 2011).

\footnotetext{
${ }^{8}$ Históricamente ha sido considerada una región periférica al eje central de producción del país, presentando una economía débil por una conjunción de factores: pocas industrias; bajo nivel de remuneraciones; un empresariado marcadamente tradicional con un fuerte predominio de PyMEs, con niveles de tecnología reducidos y predominio de agroindustrias y actividades comerciales de bajos niveles de calificación.

${ }^{9}$ La definición de juventud de la Organización de Naciones Unidas (ONU), a la cual suscribe la Oficina Internacional del Trabajo (OIT), contempla a la juventud como el grupo poblacional entre los 15 y 24 años de edad (la europea se extiende hasta los 29 años), discriminando dos subgrupos 15 a 18 años (jóvenes adolescentes) y de 19 a 24 años (jóvenes adultos). Esta distinción se encuentra sustentada en un proceso ideal que ambos grupos deberían establecer con dos mundos: educación y trabajo, siendo deseable que los jóvenes adolescentes permanezcan en el sistema educativo formal y que los jóvenes adultos realicen un ingreso ordenado y paulatino al mercado de trabajo (Balardini, 2012).
} 
Realizamos un abordaje cuantitativo con el objeto de conocer su situación en el mercado laboral de los aglomerados Gran Resistencia y Corrientes ${ }^{10}$. Los datos que se presentan resultan del análisis de las bases de usuarios (EPH) del $4^{\circ}$ trimestre de los años 2010 y 2013 , cuyo procesamiento fue realizado utilizando el software estadístico SPSS. Se consideran variables socio demográficas (sexo, situación conyugal, posición frente al hogar), educativas (nivel de escolarización alcanzado) y laborales (principales tasas del mercado: actividad, empleo, desempleo; inactividad; caracterización de las ocupaciones por ramas, niveles de calificación, intensidad y registración).

\section{CARACTERIZACIÓN GENERAL DEL GRAN RESISTENCIA Y CORRIENTES}

En este punto se presentan los datos analizados de la EPH que nos permitieron reconocer algunas condiciones estructurales en las que se construyen las trayectorias de la población juvenil antes mencionada. Si bien el análisis se centra en la caracterización de dichos aglomerados y del grupo de jóvenes, primeramente se presentan algunos datos correspondientes a la Población Económicamente Activa (PEA) de la región del Nordeste Argentino (NEA) y del Gran Resistencia y Corrientes, a fin de revisar la vinculación de los indicadores de la población juvenil con las tendencias generales del mercado.

\subsection{ALGUNOS INDICADORES DE LA PEA A NIVEL REGIONAL NEA}

En cuanto nivel educativo, en el período comprendido entre el 2010-2013 (ver Tabla $N^{o}$ 1), se observa que la mayor parte de las personas del NEA tiene estudios secundarios completos o incompletos. Al mismo tiempo se advierte un mejoramiento de estos indicadores ya que tanto a nivel regional, como en cada uno de los aglomerados en particular, existe un incremento del porcentaje de personas que lograron completar se estudios secundarios.

\footnotetext{
${ }^{10}$ Resulta importante aclarar que EPH efectúa sus mediciones solo considerando los principales aglomerados urbanos de cada una de las provincias, dejando de este modo afuera la posibilidad de describir la situación de las poblaciones rurales. No obstante, en el Gran Resistencia -integrado por la ciudad capital Resistencia, Barranqueras, Puerto Vilelas y Fontana- y Corrientes Capital, habitan entre el 35 y el $40 \%$ de la población total de cada provincia.
} 
Tabla No 1: Población total según Nivel Educativo. Región NEA, Aglomerados Gran Resistencia y Corrientes. $4^{\text {to. }}$ Trimestre 2010 y 2013.

\begin{tabular}{|c|c|c|c|c|c|c|}
\hline \multirow{2}{*}{} & \multicolumn{2}{|c|}{ NEA } & Gran Resistencia & \multicolumn{2}{c|}{ Corrientes } \\
\cline { 2 - 7 } & $\mathbf{2 0 1 0}$ & $\mathbf{2 0 1 3}$ & $\mathbf{2 0 1 0}$ & $\mathbf{2 0 1 3}$ & $\mathbf{2 0 1 0}$ & $\mathbf{2 0 1 3}$ \\
\hline Primaria Incompleta & $20,3 \%$ & $17,6 \%$ & $21,3 \%$ & $16,4 \%$ & $17,8 \%$ & $16,4 \%$ \\
\hline Primaria Completa & $12,5 \%$ & $13,4 \%$ & $14,4 \%$ & $16,4 \%$ & $10,0 \%$ & $11,5 \%$ \\
\hline Secundaria Incompleta & $21,9 \%$ & $20,6 \%$ & $22,0 \%$ & $22,5 \%$ & $21,2 \%$ & $17,4 \%$ \\
\hline Secundaria Completa & $15,1 \%$ & $18,2 \%$ & $15,1 \%$ & $18,0 \%$ & $15,3 \%$ & $19,2 \%$ \\
\hline Universitaria Incompleta & $10,4 \%$ & $10,9 \%$ & $9,6 \%$ & $9,9 \%$ & $13,7 \%$ & $13,7 \%$ \\
\hline Universitaria Completa & $8,4 \%$ & $8,3 \%$ & $7,1 \%$ & $6,3 \%$ & $10,6 \%$ & $10,8 \%$ \\
\hline Sin Instrucción & $11,4 \%$ & $11,0 \%$ & $10,5 \%$ & $10,6 \%$ & $11,3 \%$ & $11,0 \%$ \\
\hline
\end{tabular}

Fuente: elaboración propia en base a los datos de la EPH Continua, INDEC, $4^{\text {to. }}$ trimestre 2010 y 2013

En relación a los principales indicadores del mercado laboral, la tendencia a nivel de la Región NEA, y de los Aglomerados Resistencia y Corrientes, muestran un leve crecimiento de las tasas de actividad y empleo entre 2010 y 2013 (ver Tabla $\mathrm{N}^{\circ}$ 2), no obstante se observan en Resistencia menores guarismos que Corrientes en dichas tasas (esta diferencia es significativa ya que se acerca a los 10 puntos porcentuales). La Región NEA asimismo, presenta los índices más bajos de desempleo en comparación con otras regiones ${ }^{11} \mathrm{y}$, en el análisis de sus aglomerados, se visualizan también algunas diferencias ya que Corrientes evidencia un crecimiento de esta tasa en el período y en Gran Resistencia disminuye, registrando en ambos períodos valores cercanos al "pleno empleo". Esta situación pone de manifiesto una problemática que caracteriza al mercado de trabajo regional, que en lugar de significar un mejoramiento de los indicadores laborales, reflejan el reducido porcentaje de la población que trabaja y que busca trabajo ${ }^{12}$. Al respecto Golovanevsky $(2012,2013)$ lo describe como "una suerte de mercado de trabajo que implota en lugar de explotar”, manifestando las características de una economía deprimida, incapaz de absorber la mano de obra que año a año podría incorporarse al mercado de trabajo.

Tabla No 2: Principales Tasas del Mercado laboral según Región NEA, Aglomerado Gran Resistencia y Corrientes. $4^{\text {to. }}$ Trimestre 2010 y 2013.

\begin{tabular}{|c|c|c|c|c|c|c|}
\hline & \multicolumn{2}{|c|}{ NEA } & Gran Resistencia & \multicolumn{2}{c|}{ Corrientes } \\
\cline { 2 - 7 } & $\mathbf{2 0 1 0}$ & $\mathbf{2 0 1 3}$ & $\mathbf{2 0 1 0}$ & $\mathbf{2 0 1 3}$ & $\mathbf{2 0 1 0}$ & $\mathbf{2 0 1 3}$ \\
\hline Tasa de Actividad & $47,5 \%$ & $48,3 \%$ & $41,6 \%$ & $41,4 \%$ & $51,3 \%$ & $52,5 \%$ \\
\hline Tasa de Empleo & $46,1 \%$ & $47,3 \%$ & $41,2 \%$ & $41,1 \%$ & $49,4 \%$ & $50,2 \%$ \\
\hline Tasa de Desempleo & $3,0 \%$ & $2,2 \%$ & $0,9 \%$ & $0,6 \%$ & $3,7 \%$ & $4,4 \%$ \\
\hline
\end{tabular}

Fuente: elaboración propia en base a los datos de la EPH Continua, INDEC, $4^{\text {to. }}$ trimestre 2010 y 2013

\footnotetext{
${ }^{11} \mathrm{R}$ Los índices de desempleo en otras regiones del país para el 2010: GBA $8 \%$; Cuyo 4,2\%; NOA 6,6\%; Pampeana 7,6\%; Patagonia 5,3\%. En 2013: GBA 6,5\%; Cuyo 4,9\%; NOA 5,5\%; Pampeana7,7\%; Patagonia 5,5\%.

${ }^{12}$ La región NEA muestra tasas de actividad más bajas en comparación con otras regiones del país para 2010: GBA 61,2\%; Cuyo 53,5\%; NOA 56,3\%; Pampeana 57,5 \%; Patagónica 58,3\%. En tanto que para el 2013 GBA 60,9\%; Cuyo 55\%; NOA 55,7\%; Pampeana 58,3 \%; Patagónica 59,1\%; estas menores tasas de actividad se traducen en menores tasas de desocupación.
} 
En relación con el señalamiento anterior, los datos de la condición de actividad de la región y de los aglomerados muestran un escenario llamativo, ya que cerca de la mitad de la PEA manifiesta encontrarse inactivo, situación que, además, presenta una tendencia mínima a la baja entre los períodos considerados (ver Tabla $N^{o} 3$ ); no obstante es acentuada la diferencia entre los aglomerados.

Tabla $\mathrm{N}^{\circ}$ 3: Población de 14 años y más según Condición de Actividad.

Región NEA, Aglomerado Gran Resistencia y Corrientes. $4^{\text {to. }}$ Trimestre 2010 y 2013.

\begin{tabular}{|c|c|c|c|c|c|c|}
\hline & \multicolumn{2}{|c|}{ NEA } & \multicolumn{2}{c|}{ Gran Resistencia } & \multicolumn{2}{c|}{ Corrientes } \\
\cline { 2 - 7 } & $\mathbf{2 0 1 0}$ & $\mathbf{2 0 1 3}$ & $\mathbf{2 0 1 0}$ & $\mathbf{2 0 1 3}$ & $\mathbf{2 0 1 0}$ & $\mathbf{2 0 1 3}$ \\
\hline Ocupado & $46,1 \%$ & $47,2 \%$ & $41,2 \%$ & $41,1 \%$ & $51,3 \%$ & $52,5 \%$ \\
\hline Desocupado & $1,4 \%$ & $1,1 \%$ & $0,4 \%$ & $0,2 \%$ & $1,9 \%$ & $2,3 \%$ \\
\hline Inactivo & $52,4 \%$ & $51,6 \%$ & $58,4 \%$ & $58.4 \%$ & $48.6 \%$ & $47,5 \%$ \\
\hline Total & $100 \%$ & $100 \%$ & $100 \%$ & $100 \%$ & $100 \%$ & $100 \%$ \\
\hline
\end{tabular}

Fuente: elaboración propia en base a los datos de la EPH Continua, INDEC, $4^{\text {to. }}$ trimestre 2010 y 2013

En cuanto a las situaciones que reflejan la inactividad (ver Tabla $N^{o} 4$ ) se observa que mayoritariamente se trata de estudiantes, mostrando variaciones a la baja entre períodos y aglomerados, siendo esta situación más notoria en Corrientes. Asimismo, es similar el incremento, en valores porcentuales, de quienes se encuentran inactivos por su condición de jubilado/pensionado entre períodos y aglomerados. Situación contraria se manifiesta en quienes tienen la condición de ama de casa, dado que en Resistencia se produce una baja entre períodos y en Corrientes un aumento. La categoría “otros” en la que se incluyen a personas que manifiestan que no estudian, no trabajan y no manifiestan intención de hacerlo, se mantiene en Resistencia, en tanto que en Corrientes es menor el guarismo y disminuye entre 2010 y 2013.

Tabla $\mathrm{N}^{\circ}$ 4: Población de 14 años y más según Condición de Inactividad. Aglomerado Gran Resistencia y Corrientes. $4^{\text {to. }}$ Trimestre 2010 y 2013.

\begin{tabular}{|c|c|c|c|c|}
\hline \multirow{2}{*}{} & \multicolumn{2}{|c|}{ Gran Resistencia } & \multicolumn{2}{c|}{ Corrientes } \\
\cline { 2 - 5 } & $\mathbf{2 0 1 0}$ & $\mathbf{2 0 1 3}$ & $\mathbf{2 0 1 0}$ & $\mathbf{2 0 1 3}$ \\
\hline Jubilado/pensionado & $25,1 \%$ & $\mathbf{2 8 , 3} \%$ & $23,1 \%$ & $26,1 \%$ \\
\hline Rentista & $0,2 \%$ & - & $0,5 \%$ & $0,7 \%$ \\
\hline Estudiante & $35,8 \%$ & $34,7 \%$ & $40,7 \%$ & $36,5 \%$ \\
\hline Ama de casa & $24,8 \%$ & $23,0 \%$ & $23,8 \%$ & $27,1 \%$ \\
\hline Discapacitado & $0,8 \%$ & $0,1 \%$ & $0,3 \%$ & $1,3 \%$ \\
\hline Otros & $13,3 \%$ & $13,9 \%$ & $11,6 \%$ & $8,3 \%$ \\
\hline Total & $100 \%$ & $100 \%$ & $100 \%$ & $100 \%$ \\
\hline
\end{tabular}

Fuente: elaboración propia en base a los datos de la EPH Continua, INDEC, $4^{\text {to. }}$ trimestre 2010 y 2013

Hasta aquí, los datos muestran el mejoramiento en ciertos indicadores para la PEA regional y en los aglomerados considerados, como así también la persistencia de otros guarismos poco favorables que describen a esta zona del país. 


\section{CARACTERIZACIÓN DE LA POBLACIÓN JUVENIL DE 18 Y 24 AÑOS DEL GRAN RESISTENCIA Y CORRIENTES}

De acuerdo con los datos del Censo Nacional del año 2010, Gran Resistencia mostraba una población de 390.874 habitantes, de los cuales 53.175 eran jóvenes de 18 a 24 años ${ }^{13}$ $(13,6 \%)$, en tanto que en Corrientes de una población de $358.223,51.911^{14}(14,5 \%)$ corresponden al grupo en estudio. En los siguientes apartados realizamos una caracterización sociodemográfica, educativa y laboral de la población juvenil (18-24 años) a partir de datos provenientes de la Encuesta Permanente de Hogares (EPH) ${ }^{15}$, específicamente, de los aglomerados Gran Resistencia y Corrientes.

\subsection{CARACTERIZACIÓN SOCIODEMOGRÁFICA}

La distribución según sexo indica que en ambos aglomerados, en el año 2013, había una mayor proporción de varones. Si bien las proporciones son equilibradas, identificamos que esta distribución difiere de la población total (donde los valores son inversos).Se observa además que entre los años 2010 y 2013 se produjo un incremento del porcentaje de varones dentro de este grupo y de manera más pronunciada en Corrientes.

En relación con la situación conyugal la mayoría se encuentran solteros. En el año 2013 sólo 2 de cada 10 jóvenes se encontraban casados o unidos. Sin embargo, en ambos aglomerados se produjo, en el período analizado, un incremento cercano a los 3 puntos porcentuales de quienes revisten este estado. Por otra parte, si bien estos valores no son muy elevados, el NEA, junto con la Patagonia y Gran Buenos Aires son las regiones que muestran los porcentajes más altos en esta variable.

La variable antes mencionada tiene un correlato con la medición de la relación de parentesco en el hogar (ver Tabla $N^{\circ}$ 5). La mayoría de los jóvenes se ubican en la categoría de "hijos". No obstante y si bien los valores no son muy elevados, en el año 2013, un porcentaje no despreciable de jóvenes del Gran Resistencia y Corrientes, respectivamente, eran "Jefes de Hogar”. Respecto a esta variable, se puede decir también que si bien la mayoría de los jóvenes jefes de hogar poseen edades superiores a los 21 años, resulta significativo que un porcentaje importante de ellos, en el año 2010, tenían entre 18 y 20 años, siendo aún más notoria la cifra

${ }^{13}$ Varones $26.499(49,8 \%)$ y mujeres $26.675(50,2 \%)$.

${ }^{14}$ Varones $25.032(48,2 \%)$ y mujeres $26.879(51,8 \%)$.

${ }^{15}$ Se procesaron datos correspondientes al $4^{\circ}$ trimestre de los años 2010 y 2013. 
en el aglomerado del Gran Resistencia. A la vez, en el 2013, se evidencia un incremento de los jóvenes jefes de hogar, y especialmente en la franja etaria mencionada anteriormente; por el contrario en las edades más altas se aprecia una disminución de los jóvenes jefes de hogar.

Tabla $\mathrm{N}^{\circ}$ 5: Población de jóvenes de 18 a 24 según relación de parentesco. Aglomerados Gran Resistencia y Corrientes. $4^{\text {to. }}$ Trimestre 2010 y 2013.

\begin{tabular}{|c|c|c|c|c|}
\hline & \multicolumn{2}{|c|}{ Gran Resistencia } & \multicolumn{2}{c|}{ Corrientes } \\
\cline { 2 - 5 } & $\mathbf{2 0 1 0}$ & $\mathbf{2 0 1 3}$ & $\mathbf{2 0 1 0}$ & $\mathbf{2 0 1 3}$ \\
\hline Jefe & $10,70 \%$ & $13 \%$ & $10,90 \%$ & $17,30 \%$ \\
\hline Cónyuge/Pareja & $5,90 \%$ & $3,90 \%$ & $6,40 \%$ & $4,90 \%$ \\
\hline Hijo/Hijastro & $70,70 \%$ & $65,50 \%$ & $60,80 \%$ & $58,10 \%$ \\
\hline Yerno/Nuera & $0 \%$ & $6,30 \%$ & $5,20 \%$ & $3,40 \%$ \\
\hline Nieto & $6 \%$ & $8,90 \%$ & $6,40 \%$ & $3,90 \%$ \\
\hline Hermano & $1,90 \%$ & $0,70 \%$ & $5,20 \%$ & $4,60 \%$ \\
\hline Otros familiares & $2,20 \%$ & $1,10 \%$ & $0,90 \%$ & $7,40 \%$ \\
\hline No familiares & $0,60 \%$ & $0,60 \%$ & $4,20 \%$ & $0,30 \%$ \\
\hline Total & $100 \%$ & $100 \%$ & $100 \%$ & $100 \%$ \\
\hline
\end{tabular}

Fuente: elaboración propia en base a los datos de la EPH Continua, INDEC, $4^{\text {to. }}$ trimestre 2010 y 2013.

$\mathrm{Si}$ a los valores anteriores se suman quienes aparecen como "cónyuges" encontramos un grupo importante de jóvenes que, por su posición en el hogar, asumen roles diferentes al resto en cuanto al mantenimiento y reproducción de las unidades familiares y, por lo mismo, es esperable que tenga un comportamiento particular en relación con el mundo del trabajo.

En relación con los movimientos migratorios de este grupo las variables referidas al lugar de nacimiento y de residencia hace 5 años permiten identificar algunas de sus particularidades. Así, 9 de cada 10 jóvenes residen en las localidades estudiadas hace más de 5 años. Esta proporción es bastante similar en ambos aglomerados y en ambos períodos. Sin embargo no todos ellos nacieron allí. La Tabla $\mathrm{N}^{\circ} 6$ muestra la existencia de una cantidad no despreciable de jóvenes que nacieron en otras localidades o en otras provincias. Tales indicadores son un poco más elevados en Corrientes donde, por ejemplo en el año 2013, el 10,4 \% de los jóvenes encuestados mencionó haber nacido en otra localidad de la provincia y el 12, $5 \%$ en otra provincia.

Si bien la EPH no registra el momento puntual en que se produce el movimiento migratorio, los datos disponibles podrían estar indicando que la movilidad en el territorio de este grupo obedecería fundamentalmente a motivos vinculados con la continuidad de sus estudios superiores (terciarios y/o universitarios), laborales y/o con cambios de residencia de su familia de origen. Además, otro dato que nos parece relevante es que son pocos los migrantes que provienen de otros países y, en todos los casos, residen en la localidad hace más de 5 años. Esto indicaría que, a diferencia de otras regiones no constituye una problemática (social/cultural) reciente ni de peso a ser considerada desde los dispositivos orientados a este sector. 
Tabla Nº 6: Población de jóvenes de 18 a 24 años según Lugar de Nacimiento Aglomerados Gran Resistencia y Corrientes. 4to. Trimestre de 2010 y 2013.

\begin{tabular}{|c|c|c|c|c|}
\hline & \multicolumn{2}{|c|}{ Gran Resistencia } & \multicolumn{2}{c|}{ Corrientes } \\
\cline { 2 - 5 } & $\mathbf{2 0 1 0}$ & $\mathbf{2 0 1 3}$ & $\mathbf{2 0 1 0}$ & $\mathbf{2 0 1 3}$ \\
\hline En esta localidad & $85,3 \%$ & $82,6 \%$ & $75,4 \%$ & $76,7 \%$ \\
\hline En otra localidad & $6,4 \%$ & $9,9 \%$ & $11,2 \%$ & $10,4 \%$ \\
\hline En otra provincia & $7,7 \%$ & $6,7 \%$ & $13,4 \%$ & $12,5 \%$ \\
\hline En otro país & $0,6 \%$ & $0,7 \%$ & - & $0,4 \%$ \\
\hline Total & $100,0 \%$ & $100,0 \%$ & $100,0 \%$ & $100,0 \%$ \\
\hline
\end{tabular}

Fuente: elaboración propia en base a los datos de la EPH Continua, INDEC, $4^{\text {to. }}$ trimestre 2010 y 2013

\subsection{CARACTERIZACIÓN DE LA SITUACIÓN EDUCATIVA}

En ambos aglomerados el porcentaje de jóvenes que no finalizaron estudios secundarios es elevado. Es aún mayor en Gran Resistencia donde 3 de cada 10, en el año 2013, se encontraban en esta situación -en tanto que esta proporción se reduce a 2 cada 10 en Corrientes- (ver tabla $N^{\circ} 7$ ).

Tabla $\mathrm{N}^{\circ}$ 7: Población de Jóvenes de 18 a 24 años según Nivel Educativo. Aglomerados Gran Resistencia y Corrientes. 4to. trimestre de 2010 y 2013.

\begin{tabular}{|c|c|c|c|c|}
\hline & \multicolumn{2}{|c|}{ Gran Resistencia } & \multicolumn{2}{|c|}{ Corrientes } \\
\cline { 2 - 4 } & $\mathbf{2 0 1 0}$ & $\mathbf{2 0 1 3}$ & $\mathbf{2 0 1 0}$ & $\mathbf{2 0 1 3}$ \\
\hline Primaria Incompleta (incluye educación especial) & $2,2 \%$ & $3,4 \%$ & $2,7 \%$ & $4,4 \%$ \\
\hline Primaria Completa & $5,9 \%$ & $3,5 \%$ & $6,1 \%$ & $8,0 \%$ \\
\hline Secundaria Incompleta & $36,7 \%$ & $31,9 \%$ & $27,7 \%$ & $21,7 \%$ \\
\hline Secundaria Completa & $19,6 \%$ & $24,3 \%$ & $21,3 \%$ & $18,6 \%$ \\
\hline Superior Universitaria Incompleta & $32,6 \%$ & $35,9 \%$ & $37,9 \%$ & $44,3 \%$ \\
\hline Superior Universitaria Completa & $2,6 \%$ & $0,9 \%$ & $3,8 \%$ & $2,9 \%$ \\
\hline Sin instrucción & $0,4 \%$ & - & $0,6 \%$ & - \\
\hline Total & $100 \%$ & $100 \%$ & $100 \%$ & $100 \%$ \\
\hline
\end{tabular}

Fuente: elaboración propia en base a los datos de la EPH Continua, INDEC, $4^{\text {to. }}$ trimestre 2010 y 2013.

El análisis de la distribución según edades de este grupo indica, además, que es elevado el porcentaje de aquellos que poseen más de 18 o 19 años (edad esperable de finalización de dicho trayecto formativo) que se encuentran en dicha situación. No obstante, en ambos aglomerados se observa una significativa reducción de los jóvenes que pertenecen a este grupo entre los años 2010 y 2013. Estos datos podrían estar indicando un impacto positivo de los programas y planes con componentes orientados a la terminalidad educativa en este nivel y, a su vez, dan cuenta del incremento cada vez más pronunciado de los “jóvenes” en la modalidad de Educación de Jóvenes y Adultos, dentro de los organismos educativos del Sistema Formal. 
En consecuencia, también se registra un mejoramiento del porcentaje de jóvenes que continúan sus estudios en el nivel superior universitario, lo hayan completado o no (ver Tabla $\mathrm{N}^{\circ} 7$ ). En Corrientes, además de ser un poco más elevada la cantidad de jóvenes que integran este grupo, también es más pronunciado el incremento del mismo entre el 2010 y 2013. Si bien la Universidad Nacional del Nordeste (UNNE) tiene ofertas educativas en ambas localidades, esta diferencia quizá pueda ser explicada por la existencia en Corrientes de algunas carreras tradicionales que tienen una matrícula más elevada (como Abogacía y Medicina) u otras específicas (que no se ofrecen en otras universidades públicas ni privadas del norte argentino) como Ciencias Agrarias y Veterinaria. Al mismo tiempo la mayor presencia de Universidades Privadas en Corrientes, podrían explicar esta diferencia.

\subsection{SITUACIÓN LABORAL}

El análisis de los indicadores generales del mercado de trabajo para la población juvenil de la población joven (18-24 años) muestra, en términos generales un comportamiento similar al de la población total que describimos en el apartado inicial, a saber:

* Comparando con otras regiones (ver tabla $N^{\circ} 8$ ), los jóvenes del NEA son los que muestran las tasas más bajas de actividad, con una pronunciada diferencia de entre 10 y 30 puntos en relación con las otras. En relación con esto, es también la región que presenta las tasas más bajas de empleo y de desocupación.

Tabla 8: Jóvenes de 18 a 24 años según principales tasas del mercado de trabajo por Regiones, $4^{\text {to. }}$ Trimestre 2010 y 2013

\begin{tabular}{|c|c|c|c|c|c|c|}
\hline \multirow{2}{*}{ Regiones } & \multicolumn{2}{|c|}{ ACTIVIDAD } & \multicolumn{2}{c|}{ EMPLEO } & \multicolumn{2}{c|}{ DESOCUPACIÓN } \\
\cline { 2 - 7 } & $\mathbf{2 0 1 0}$ & $\mathbf{2 0 1 3}$ & $\mathbf{2 0 1 0}$ & $\mathbf{2 0 1 3}$ & $\mathbf{2 0 1 0}$ & $\mathbf{2 0 1 3}$ \\
\hline Gran Buenos Aires & $65,20 \%$ & $60,79 \%$ & $52,06 \%$ & $50,55 \%$ & $20,22 \%$ & $16,83 \%$ \\
\hline NOA & $44,80 \%$ & $43,80 \%$ & $37,43 \%$ & $36,25 \%$ & $8,96 \%$ & $17,33 \%$ \\
\hline NEA & $31,70 \%$ & $36,30 \%$ & $29,78 \%$ & $34,07 \%$ & $2,87 \%$ & $6,37 \%$ \\
\hline Cuyo & $40,00 \%$ & $44,27 \%$ & $36,23 \%$ & $37,69 \%$ & $4,82 \%$ & $14.86 \%$ \\
\hline Pampeana & $49,90 \%$ & $49,86 \%$ & $39,19 \%$ & $38,99 \%$ & $10,50 \%$ & $21,79 \%$ \\
\hline Patagónica & $47,80 \%$ & $44,12 \%$ & $37,23 \%$ & $36,69 \%$ & $8,21 \%$ & $16,83 \%$ \\
\hline Total & $55,10 \%$ & $53,01 \%$ & $44,64 \%$ & $43,73 \%$ & $10,26 \%$ & $17,44 \%$ \\
\hline
\end{tabular}

Fuente: elaboración propia en base a los datos de la EPH Continua, INDEC, $4^{\text {to. }}$ trimestre 2010 y 2013.

* Al interior de la región NEA también identificamos algunas diferencias. Gran Resistencia es el aglomerado que posee la tasa más baja de actividad, con más de 10 puntos porcentuales de diferencia con Corrientes, lo cual tiene su correlato en las tasas más bajas de empleo (cerca de 8 puntos de diferencia) y de desocupación (ver Tabla $N^{\circ} 9$ ). 
Tabla Nº 9: Población de jóvenes 18-24 años según principales Tasas del mercado de trabajo. Región NEA y Aglomerados Gran Resistencia y Corrientes. $4^{\text {to. }}$ Trimestre 2010 y 2013

\begin{tabular}{|c|c|c|c|c|c|c|}
\hline & \multicolumn{2}{|c|}{ Total Región } & \multicolumn{2}{c|}{ Corrientes } & \multicolumn{2}{c|}{ G. Resistencia } \\
\cline { 2 - 7 } & $\mathbf{2 0 1 0}$ & $\mathbf{2 0 1 3}$ & $\mathbf{2 0 1 0}$ & $\mathbf{2 0 1 3}$ & $\mathbf{2 0 1 0}$ & $\mathbf{2 0 1 3}$ \\
\hline Tasa de Actividad & $31,7 \%$ & $36,3 \%$ & $31,9 \%$ & $38,8 \%$ & $22,2 \%$ & $23,8 \%$ \\
\hline Tasa de Empleo & $37,4 \%$ & $36,2 \%$ & $30 \%$ & $35,1 \%$ & $21,8 \%$ & $23,8 \%$ \\
\hline Tasa de Desocupación & $2,8 \%$ & $6,4 \%$ & $6 \%$ & $9,6 \%$ & $1,7 \%$ & $0 \%$ \\
\hline Tasa de Subocupaciòn Horaria Demandante & $3,8 \%$ & $5,1 \%$ & $2,8 \%$ & $3,7 \%$ & $8,9 \%$ & $6,27 \%$ \\
\hline Tasa de Subocupación Horaria No Demandante & $0,3 \%$ & $2,7 \%$ & $0 \%$ & $0 \%$ & $1,6 \%$ & $0 \%$ \\
\hline
\end{tabular}

Fuente: elaboración propia en base a los datos de la EPH Continua, INDEC, $4^{\text {to. }}$ trimestre 2010 y 2013.

Por su parte, los datos referidos a condición de actividad (ver Tabla $\mathrm{N}^{\circ} 10$ ) muestran que:

* Se produjo un incremento del porcentaje de jóvenes ocupados en ambos aglomerados (esto es más pronunciado en Corrientes donde hay 5 puntos de diferencia entre ambos años, y en el caso del Gran Resistencia sólo de 2).

* Se redujo el porcentaje de inactivos en ambos aglomerados (aunque las mismas superan el 60\% en Corrientes y el 70\% en Resistencia), observándose una reducción más pronunciada en la primera. A su vez Gran Resistencia muestra en ambos períodos una cantidad mucho mayor de jóvenes inactivos (en el 2010 esta diferencia era de 9.7 puntos y en el 2013 de 15 puntos).

* Es llamativo el dato de la inexistencia de desocupados en el Gran Resistencia.

Tabla $N^{\circ}$ 10: Población de Jóvenes de 18 a 24 años según Condición de Actividad. Aglomerados Gran Resistencia y Corrientes. 4to. Trimestre 2010 y 2013.

\begin{tabular}{|c|c|c|c|c|}
\hline & \multicolumn{2}{|c|}{ Gran Resistencia } & \multicolumn{2}{c|}{ Corrientes } \\
\cline { 2 - 5 } & $\mathbf{2 0 1 0}$ & $\mathbf{2 0 1 3}$ & $\mathbf{2 0 1 0}$ & $\mathbf{2 0 1 3}$ \\
\hline Ocupado & $21,80 \%$ & $23,80 \%$ & $30 \%$ & $35,10 \%$ \\
\hline Desocupado & $0,40 \%$ & - & $1,90 \%$ & $3,70 \%$ \\
\hline Inactivo & $77,80 \%$ & $76,20 \%$ & $68,10 \%$ & $61,20 \%$ \\
\hline Total & $100 \%$ & $100 \%$ & $100 \%$ & $100 \%$ \\
\hline
\end{tabular}

Fuente: elaboración propia en base a los datos de la EPH Continua, INDEC, $4^{\text {to. }}$ trimestre 2010 y 2013.

En líneas generales podemos señalar que, pese al leve incremento de la participación laboral juvenil, anteriormente mostrábamos la mayor permanencia de los jóvenes en el sistema educativo. Esta situación plantea algunas cuestiones sobre la oferta laboral juvenil: en términos cuantitativos la baja participación laboral y en términos cualitativos que los jóvenes ingresan al mercado de trabajo con mejores niveles educacionales. Como resultado de este proceso habría una menor presión en la oferta laboral juvenil y una mayor calidad de la mano de obra de los jóvenes que acceden al mercado de trabajo, lo que tendería a favorecer la inserción laboral juvenil (OIT, 2013). Restaría analizar si dicho incremento de capacidades formativas se traducen en el mediano plazo en mejores condiciones de inserción. 


\subsubsection{SOBRE EL PERFIL DE LOS INACTIVOS:}

Como señalamos anteriormente, en ambos aglomerados, para el grupo de jóvenes de entre 18 y 24 años, se redujo en porcentaje de inactivos. La categoría de inactividad permite describir, además, la variación del perfil de los inactivos.

En ambos aglomerados la mayoría de los jóvenes inactivos se encuentran en la categoría de "estudiantes". También en ambos se redujo el porcentaje de "otros" ${ }^{16}$, aunque sigue siendo más elevado este valor en Gran Resistencia (ver Tabla $N^{\circ} 11$ ). La reducción de esta categoría se traduce a su vez en un incremento en la condición de "estudiantes" y "amas de casa”. La hipótesis aquí es que estas transformaciones obedecen al impacto de algunos programas de educación, trabajo y de la Seguridad Social (Asignación Universal por Hijos, componentes de terminalidad educativa, becas, etc.) que acompañan estos cambios.

Tabla $\mathrm{N}^{\circ}$ 11: Población de Jóvenes de 18 a 24 años según Categoría de Inactividad.

Aglomerados Gran Resistencia y Corrientes. $4^{\text {to. }}$ Trimestre 2010 y 2013.

\begin{tabular}{|c|c|c|c|c|}
\hline \multirow{2}{*}{} & \multicolumn{2}{|c|}{ Gran Resistencia } & \multicolumn{2}{c|}{ Corrientes } \\
\cline { 2 - 5 } & $\mathbf{2 0 1 0}$ & $\mathbf{2 0 1 3}$ & $\mathbf{2 0 1 0}$ & $\mathbf{2 0 1 3}$ \\
\hline Jubilado/pensionado & $1,50 \%$ & - & $1,10 \%$ & $2,50 \%$ \\
\hline Estudiante & $53,60 \%$ & $56,90 \%$ & $62,80 \%$ & $63,60 \%$ \\
\hline Ama de casa & $15,90 \%$ & $17,20 \%$ & $17,40 \%$ & $19,20 \%$ \\
\hline Discapacitado & - & - & $0,40 \%$ & - \\
\hline Otros & $29,10 \%$ & $25,90 \%$ & $18,20 \%$ & $14,80 \%$ \\
\hline Total & $100 \%$ & $100 \%$ & $100 \%$ & $100 \%$ \\
\hline
\end{tabular}

Fuente: elaboración propia en base a los datos de la EPH Continua, INDEC, $4^{\text {to. }}$ trimestre 2010 y 2013

No obstante, y en relación a esta categoría, se puede advierter que los mayores porcentajes se concentran en aquellos jóvenes con secundaria incompleta y completa, y en términos de comparación entre los períodos estudiados, se aprecia una disminución en ambos aglomerados de aquellos jóvenes con estudios secundarios completos que no estudian ni trabajan, pero, y por el contrario, se muestra un crecimiento de este grupo de jóvenes cuyo nivel educativo resulta ser el secundario incompleto. Al respecto Salvia (op. cit.) señala que uno de los

\footnotetext{
${ }^{16}$ La categoría "Otros" representa en la EPH a aquellas personas que declaran no estudiar, no trabajar, pudiendo incluir diferentes situaciones que no son relevadas con profundidad a fin de reconocer la condición real de los jóvenes. Al respecto, en un reciente artículo publicado por el portal "Jóvenes con Voz" de Organización Internacional de la Juventud (OIJ), Ernesto Rodríguez (2014) señala que al momento de realizar las encuestas de las cuales surgen los datos a partir de los cuales se "identifican" a los Jóvenes peyorativamente denominados "ni-ni"las respuestas posibles son muy pocas e inducen a pensar (a quienes son interrogados) que "trabajar" significa "estar contratados en una empresa formal" y que "estudiar" significa "estar asistiendo a clases a una escuela secundaria o a una universidad", esto es, a suponer que "trabajo" es sólo "trabajo formal" y que "estudiar" es sólo "estudiar en una escuela formal", dejando por fuera numerosas experiencias y situaciones en las cuales participan.
} 
principales motivos del mantenimiento de la inactividad juvenil se debe a la mayor dedicación exclusiva de los jóvenes a la educación formal, lo que se observa en ambos aglomerados. Esta evolución es consistente con la extensión de la cobertura de la educación media que a través de distintos regímenes organizativos del ciclo ha promovido la inclusión de muchos jóvenes al ámbito escolar (Vezza y Bertranou, op. cit.).

También en el período analizado, tuvo lugar un crecimiento de la inactividad doméstica, los cuales podrían ser leídos como resultado de procesos recuperación económica y de programas sociales, que cobran mayor notoriedad en el caso de mujeres jóvenes que se encuentran a cargo de quehaceres domésticos y del cuidado de hijos, hermanos menores u otros miembros del hogar, percibiendo a través de ellos una ayuda económica mediante programas asistenciales $^{17}$ (Salvia, op. cit.).

\subsubsection{CARACTERIZACIÓN DE LA OCUPACIÓN JUVENIL}

En relación con el tipo de establecimiento, la mayoría de los jóvenes ocupados se insertan en el sector privado. En ambos aglomerados se produjo un incremento de la participación en este sector, pero esto es mucho más pronunciado en Gran Resistencia. Es llamativo, especialmente en el caso del Gran Resistencia, la reducción del empleo público juvenil (Ver Tabla $N^{\circ} 12$ ), ya que como lo señalamos en la presentación de la problemática este sector constituye en la región uno de los tradicionales espacios de inserción laboral de la población en general.

Tabla $\mathrm{N}^{\circ}$ 12: Jóvenes ocupados asalariados de 18 a 24 años según tipo de establecimiento.

Aglomerados Gran Resistencia y Corrientes. $4^{\text {to. }}$ Trimestre 2010 y 2013.

\begin{tabular}{|c|c|c|c|c|}
\hline & \multicolumn{2}{|c|}{ Gran Resistencia } & \multicolumn{2}{c|}{ Corrientes } \\
\cline { 2 - 5 } & $\mathbf{2 0 1 0}$ & $\mathbf{2 0 1 3}$ & $\mathbf{2 0 1 0}$ & $\mathbf{2 0 1 3}$ \\
\hline Estatal & $22,50 \%$ & $7,50 \%$ & $16,80 \%$ & $35,10 \%$ \\
\hline Privado & $77,50 \%$ & $88,70 \%$ & $83,20 \%$ & $3,70 \%$ \\
\hline De otro tipo & - & $3,90 \%$ & - & $61,20 \%$ \\
\hline Total & $100 \%$ & $100 \%$ & $100 \%$ & $100 \%$ \\
\hline
\end{tabular}

Fuente: elaboración propia en base a los datos de la EPH Continua, INDEC, $4^{\text {to. }}$ trimestre 2010 y 2013.

\footnotetext{
${ }_{17}$ Si bien Davolos (2013) en un estudio sobre condicionantes de participación en el mercado de trabajo de perceptoras de la Asignación Universal por Hijo señala que la percepción de este derecho no implica necesariamente la entrada en la inactivad o reducción del tiempo dedicado al trabajo, las formas de vinculación y la dedicación al trabajo remunerado están fuertemente enlazadas por una distribución desigual del cuidado que coloca a estas madres en un lugar casi exclusivo.
} 
Los datos sobre las ramas de actividades permiten interpretar mejor estas variaciones, describir los sectores donde se insertan los jóvenes e identificar cuales fueron más dinámicos, en estos años, en la generación de puestos de trabajo.

Como es esperable, debido a las características generales de la estructura del mercado de trabajo de ambos aglomerados, la inserción de jóvenes en el sector primario y en la industria manufacturera es muy baja o inexistente ${ }^{18}$.

$\mathrm{Al}$ igual que en otras regiones las ramas de actividad en las que se registra una mayor ocupación juvenil son la construcción, los comercios, el sector servicios y el servicio doméstico en hogares privados (Ver tabla $N^{\circ} 13$ ). El empleo en estas actividades en muchos casos suele ser de baja calificación, requiriendo menos experiencia y credenciales que otros, por lo cual muchas trayectorias ocupacionales de los jóvenes se inician en ellos (Golovanevsky, op.cit.).

Sin embargo, la comparación de la evolución temporal (2010-2013) de esta variable así como entre los aglomerados estudiados, permiten hacer algunas consideraciones y distinciones interesantes. El sector construcciones, fue el que mostró mayor dinamismo en la generación de empleo ya que en ambos aglomerados, entre los años estudiados, tuvo un incremento cercano al 100\%. Aproximadamente el 25\% de los jóvenes que trabajan lo hacen en este sector, empleando mayoritariamente a varones.

El sector comercio, se ubica en el segundo orden entre las que mayores ocupaciones ofrecen ${ }^{19}$ pero muestra comportamientos diferentes el Gran Resistencia y en Corrientes. Mientras que en el primero sufrió una leve reducción en el segundo prácticamente se duplicó ${ }^{20}$. Aun así, Resistencia, quizás por su ya tradicional e intensa actividad comercial continúa mostrando una diferencia superior de más de 6 puntos porcentuales. Se diferencia también en términos de resultar un sector que emplea más mano de obra femenina que en el aglomerado de Corrientes.

La rama de servicios, por su parte, constituye otro gran sector en la que los jóvenes logran insertarse aunque el mismo incluye una alta heterogeneidad respecto a los tipos de actividades. En el caso del Gran Resistencia, la ocupación de jóvenes en los servicios de transporte, almacenaje y comunicaciones así como en los inmobiliarios, financieros y empresariales se produjo un notable incremento en los últimos años. En paralelo se produjo una reducción significativa en los servicios sociales, comunitarios y personales (entre ellos en la Administración Pública y en las Fuerzas Armadas y Policía).

\footnotetext{
${ }^{18}$ No obstante llama la atención que, en el 2013, las EPH no registran ningún joven ocupado en el sector industrial debido a que en los últimos años se produjo la apertura de algunas instalaciones fabriles en el Gran Resistencia.

${ }^{19}$ A su vez, dentro de esta rama, los tipos de comercios con mayor cantidad de trabajadores jóvenes son los vinculados a la venta de mercaderías, alimentos, bebida y tabaco.

20 En el año 2013 en Corrientes hay un 4.2\% de jóvenes que aparecen registrados en "venta al por menor en puestos móviles y no realizados en tiendas".
} 
En Corrientes, por su parte, el crecimiento de la ocupación se produjo también en los servicios de transporte, almacenaje y comunicaciones pero, además, en los servicios sociales y de salud. En la Administración pública y en las fuerzas armadas y policía, a diferencia de Resistencia, se produjo también un leve incremento.

Finalmente un sector que también muestra algunas modificaciones importantes es el de las actividades de servicio doméstico en hogares privados. En ambos aglomerados se redujo de manera significativa. Debemos señalar que tradicionalmente este es un sector de inserción de mujeres (y que además son jóvenes), lo cual las expone a una situación de desventaja, ya que estos empleos son de baja calificación, alta desprotección de seguridad social y bajos ingresos. Asimismo, la variación observada en los porcentajes de ocupación en ella quizá se vincule también con los efectos de planes sociales y de seguridad social, que al asegurar un ingreso posibilitaron que muchas se retiraran como oferentes en este sector del mercado de trabajo. Con todo, es importante señalar que la ocupación en este tipo de actividad continúa siendo notablemente mayor en Corrientes y probablemente se asocie a las prácticas culturales y sociales, y el tradicional consumo de este tipo de servicio en las familias Correntinas ${ }^{21}$.

Tabla $\mathrm{N}^{\circ}$ 13. Jóvenes ocupados según actividad económica. Aglomerados Gran Resistencia y Corrientes. 4to. Trimestre 2010 y 2013

\begin{tabular}{|c|c|c|c|c|}
\hline \multirow{2}{*}{ Agricultura } & \multicolumn{2}{|c|}{ Gran Resistencia } & \multicolumn{2}{c|}{ Corrientes } \\
\cline { 2 - 5 } & $\mathbf{2 0 1 0}$ & $\mathbf{2 0 1 3}$ & $\mathbf{2 0 1 0}$ & $\mathbf{2 0 1 3}$ \\
\hline Industria & $2,1 \%$ & - & - & $1,4 \%$ \\
\hline Construcción & - & - & $4 \%$ & $1,3 \%$ \\
\hline Comercio & $12,1 \%$ & $24,4 \%$ & $13,8 \%$ & $26,3 \%$ \\
\hline Servicios & $20,7 \%$ & $19,4 \%$ & $8 \%$ & $15,8 \%$ \\
\hline Administración Publica y Defensa & $23,1 \%$ & $18,1 \%$ & $15,9 \%$ & $14,6 \%$ \\
\hline Enseñanza & $17,6 \%$ & $5 \%$ & $4 \%$ & $7,1 \%$ \\
\hline Servicio sociales & - & $2,5 \%$ & $2,4 \%$ & $2,4 \%$ \\
\hline Artes, entrenamiento y recreación & $4,7 \%$ & $5, \% 7$ & $6 \%$ & $3,5 \%$ \\
\hline Servicio doméstico & $3,6 \%$ & - & $2,8 \%$ & $6,5 \%$ \\
\hline & $9,5 \%$ & $4,9 \%$ & $24,7 \%$ & $13,4 \%$ \\
\hline
\end{tabular}

Fuente: elaboración propia en base a los datos de la EPH Continua, INDEC, 4 to. trimestre 2010 y 2013. Agrupada según Clasificación de Actividades Económicas para Encuestas Sociodemográficas del MERCOSUR-Versiones 2010 y 2011.

En cuanto a las calificaciones de las ocupaciones desempeñadas por los jóvenes en los diferentes sectores, las mismas son mayoritariamente operativas y no calificadas, apreciándose ciertas diferencias entre ambos aglomerados (ver Tabla 14). En el Gran Resistencia hubo

\footnotetext{
${ }^{21}$ Kiss (2005) señala que Corrientes se caracteriza por una estructura social altamente polarizada entre los más pobres y los más ricos, donde continúan vigentes modelos culturales patriarcales y autoritarios, que sostienen las antiguas relaciones de "vasallaje".
} 
una disminución de la ocupación de puestos no calificados y un aumento de la realización de actividades calificadas como operativas. En Corrientes, aunque también se redujo el porcentaje de actividades no calificadas, es menor que en Gran Resistencia (sólo se da en 4 puntos) y se evidencia, en cambio, un aumento importante del desempeño de tareas que requieren una calificación técnica.

Tabla No 14: Jóvenes ocupados asalariados de 18 a 24 años según calificación ocupacional. Aglomerados Gran Resistencia y Corrientes. $4^{\text {to. }}$ Trimestre 2010 y 2013. En valores relativos.

\begin{tabular}{|c|c|c|c|c|}
\hline \multirow{2}{*}{} & \multicolumn{2}{|c|}{ Gran Resistencia } & \multicolumn{2}{c|}{ Corrientes } \\
\cline { 2 - 5 } & $\mathbf{2 0 1 0}$ & $\mathbf{2 0 1 3}$ & $\mathbf{2 0 1 0}$ & $\mathbf{2 0 1 3}$ \\
\hline Profesional & $5,1 \%$ & - & $2,5 \%$ & - \\
\hline Técnico & $1,9 \%$ & - & $1,1 \%$ & $11,9 \%$ \\
\hline Operativo & $42,9 \%$ & $62,2 \%$ & $41,1 \%$ & $36,9 \%$ \\
\hline No calificado & $50,1 \%$ & $37,8 \%$ & $55,3 \%$ & $51,1 \%$ \\
\hline Total & $100,0 \%$ & $100,0 \%$ & $100,0 \%$ & $100,0 \%$ \\
\hline
\end{tabular}

Fuente: elaboración propia en base a los datos de la EPH Continua, INDEC, 4to. trimestre 2010 y 2013

Respecto a la intensidad de la ocupación, entre el año 2010 y 2013, se produjo un incremento notable del porcentaje de jóvenes que tienen ocupación plena en el Gran Resistencia. Ocurre lo contrario en Corrientes donde se este tipo de ocupación se redujo en casi 14 puntos. $\mathrm{Al} 2013$ los valores en ambos aglomerados son bastante próximos, sin embargo indican que: a) sólo la mitad de los ocupados poseen condiciones adecuadas de trabajo en términos de intensidad (más del 40\% mencionaron estar sobreocupados); b) en Corrientes, en los últimos años, el empeoramiento de estas condiciones se vincula justamente con el incremento de dicha sobreocupación horaria (ver Tabla $\left.N^{\mathrm{o}} 15\right)$.

Tabla $\mathrm{N}^{\circ}$ 15: Población de jóvenes ocupados según intensidad de la ocupación. Aglomerados Gran Resistencia y Corrientes. $4^{\text {to. }}$ Trimestre 2010 y 2013.

\begin{tabular}{|c|c|c|c|c|}
\hline \multirow{2}{*}{} & \multicolumn{2}{|c|}{ Gran Resistencia } & \multicolumn{2}{c|}{ Corrientes } \\
\cline { 2 - 5 } & $\mathbf{2 0 1 0}$ & $\mathbf{2 0 1 3}$ & $\mathbf{2 0 1 0}$ & $\mathbf{2 0 1 3}$ \\
\hline Subocupación horaria Demandante & $9,10 \%$ & - & $3 \%$ & $4,20 \%$ \\
\hline Subocupación horaria No Demandante & $1,60 \%$ & $6,30 \%$ & - & - \\
\hline Ocupación plena & $28,50 \%$ & $51,30 \%$ & $66,60 \%$ & $52,70 \%$ \\
\hline Sobreocupación horaria & $59,40 \%$ & $42,40 \%$ & $27,30 \%$ & $41 \%$ \\
\hline Ocupado que no trabajó en la semana & $1,50 \%$ & - & $3,10 \%$ & $2,10 \%$ \\
\hline Total & $100 \%$ & $100 \%$ & $100 \%$ & $100 \%$ \\
\hline
\end{tabular}

Fuente: elaboración propia en base a los datos de la EPH Continua, INDEC, $4^{\text {to. }}$ trimestre 2010 y 2013

En lo relativo a la precarización laboral interesa revisar cuestiones vinculadas a la registración e inseguridad en el empleo de los jóvenes. En relación con ello, se advierte que es elevado el porcentaje de jóvenes que declaran que al momento de percibir su remuneración 
"no reciben nada" (recibo de sueldo, entrega de factura, etc.) por parte de los empleadores. Los valores, en ambos períodos, muestran una situación más desfavorable para Corrientes, donde el $70 \%$ de los ocupados asalariados mencionan estar en esta situación, en tanto en el Gran Resistencia varía entre el 45 y $50 \%$.

Tabla $\mathrm{N}^{\circ}$ 16: Población de jóvenes ocupados de 18 a 24 años según niveles de formalidad laboral. Aglomerados de Gran Resistencia y Corrientes. $4^{\text {to. }}$ Trimestre 2010 y 2013. En valores relativos.

\begin{tabular}{|c|c|c|c|c|}
\hline \multirow{2}{*}{} & Gran Resistencia & \multicolumn{2}{|c|}{ Corrientes } \\
\cline { 2 - 4 } & $\mathbf{2 0 1 0}$ & $\mathbf{2 0 1 3}$ & $\mathbf{2 0 1 0}$ & $\mathbf{2 0 1 3}$ \\
\hline ...le dan recibo con sello/membrete/firma del empleador? & $36,30 \%$ & $35,50 \%$ & $23,40 \%$ & $24,90 \%$ \\
\hline ...le dan un papel/recibo sin nada? & $4,70 \%$ & $15,80 \%$ & $2,30 \%$ & $5,50 \%$ \\
\hline ...entrega una factura? & $9,80 \%$ & $3,40 \%$ & - & - \\
\hline ...no le dan ni entrega nada? & $49,10 \%$ & $45,30 \%$ & $70,70 \%$ & $69,70 \%$ \\
\hline no cobra, es trabajador sin pago, ad-honorem & - & - & $3,60 \%$ & - \\
\hline Total & $100 \%$ & $100 \%$ & $100 \%$ & $100 \%$ \\
\hline
\end{tabular}

Fuente: elaboración propia en base a los datos de la EPH Continua, INDEC, $4^{\text {to. }}$ trimestre 2010 y 2013

Si bien entre los años comparados se produjo una disminución de la cantidad jóvenes ubicados en este grupo, los valores no muestran cambios significativos ${ }^{22}$. Esto indicaría las dificultades (al menos para este grupo etario) que tendrían los programas que promueven el empleo registrado. En este sentido, según Cortez y Graña (2013) los estudios que analizan la evolución de los índices de empleo no registrado, señalan que el mismo se mantiene alto tanto en fases de crecimiento económico como de recesión, lo cual los lleva a plantear que su persistencia no obedecería a la evolución del producto, sino a características estructurales de la precariedad.

\section{REFLEXIONES FINALES}

Los procesos de inserción laboral de los jóvenes si bien presentan algunos rasgos particulares que los distinguen de otros grupos etarios, también se encuentran estrechamente vinculados a ciertas características y transformaciones del mercado laboral regional. Los aglomerados analizados, Gran Resistencia y Corrientes, se encuentran en la región (NEA) que posee los indicadores más bajos de actividad, ocupación y educación del país. A pesar de que en los

\footnotetext{
${ }^{22}$ Golovanevsky (2013) observa que si bien los niveles de precariedad laboral para la PEA se redujeron durante los últimos 10 años, siguen persistiendo situaciones de elevada vulnerabilidad particularmente entre los jóvenes ocupados, siendo el NEA una de las zonas con mayor persistencia de esta situación.
} 
últimos años se produjo un mejoramiento de ciertas condiciones socio-económicas a nivel nacional (así como de algunos indicadores del mercado de trabajo para la población general) las diferencias con otras regiones persisten y los jóvenes en particular continúan en situaciones desventajosas al momento de integrarse al mundo del trabajo. Al mismo tiempo, y a pesar de la proximidad geográfica se observan diferencias significativas en la situación de los jóvenes en ambos aglomerados urbanos.

Puntualmente, el análisis realizado de la franja etaria en el período 2010-2013 muestra que:

* Aunque en ambos aglomerados continúan observándose porcentajes elevados de jóvenes que no han finalizado estudios de nivel secundario, se visualiza el incremento de quienes se encuentran insertos en el sistema educativo, lo cual lleva a suponer el impacto favorable de programas de distinto tipo que tienen como objetivo el retorno y terminalidad de educativa de los ciclos obligatorios. Asimismo, se observa el leve incremento de jóvenes que continúan estudios de nivel superior universitario.

* En cuanto a aspectos del mercado de trabajo, se ha incrementado la cantidad de jóvenes ocupados, lo cual impactó en la reducción de la inactividad aunque con ciertas diferencias entre aglomerados, mostrando Corrientes guarismos de mayor reducción de esta situación. El alto porcentaje de inactivos lleva en el Gran Resistencia a tasas de desempleo cercanas a cero. En este marco, conforme a los datos analizados, el desempleo juvenil no representaría una problemática acuciante, sino que la misma está dada por el desaliento a su participación en el mercado de trabajo, evidenciándose en la mayor permanencia y regreso al sistema educativo.

* Los jóvenes que se encuentran ocupados lo están mayoritariamente en el sector privado de la economía, específicamente en la construcción y el comercio. La primera es una actividad de gran crecimiento en ambos aglomerados en los últimos años, y la segunda, una fuente de empleo tradicional en la región. Conjuntamente, el sector servicios y el empleo doméstico se constituyen en otros ámbitos relevantes para la inserción laboral juvenil. En relación con los sectores de inserción, los cuales se caracterizan mayoritariamente por la existencia de puestos de trabajo que no demandan elevadas calificaciones, los jóvenes se insertan fundamentalmente en ocupaciones de tipo no calificadas y operativas.

* En lo referente a ciertos aspectos de las condiciones de trabajo se visualizan algunos cambios en torno a la cantidad de horas de trabajo de los jóvenes que se vinculan al pleno empleo y la sobreocupación horaria. Por otra parte continúan presentándose altos porcentajes de jóvenes ocupados que no están registrados en los sistemas de seguridad social, siendo más grave la situación en Corrientes. Si bien en los últimos años, desde los organismos oficiales vinculados a la gestión del trabajo y el empleo, también se ha desarrollado una nueva institucionalidad que intenta promover la regularización del trabajo, su impacto positivo parece haber sido mucho menor (al menos para este grupo etario) si se lo compara con el de los programas vinculados a la terminalidad educativa y formación para el trabajo.

La descripción realizada hasta aquí resulta útil, como una caracterización contextual ge- 
neral, ya que nos permite reconocer algunas condiciones estructurales en las que se construyen las trayectorias socioeducativas y laborales de los jóvenes. Así, resulta posible considerar que los problemas de empleo juvenil no tendrían como principal sustrato la débil empleabilidad sino los límites estructurales que presenta la demanda laboral, a lo cual se agregan las desiguales condiciones materiales de origen y los diferentes entornos socioculturales.

En el mismo sentido, a partir de este estudio advertimos la importancia de avanzar en análisis situados a nivel local pero que, además, incorpore como dimensión relevante las particularidades y especificidades de sectores o ramas específicos de actividad (la organización y el contenido de los puestos de trabajo, la tecnología disponible, las políticas y prácticas de gestión de la fuerza de trabajo en las empresas, entre otros) ya que los mismos, a la vez que determinan las necesidades y demandas de formación, inciden en la construcción de trayectorias juveniles más o menos calificantes.

\section{REFERENCIAS BIBLIOGRÁFICAS}

Abeles, M. (2009) El impacto de la crisis internacional en la economía argentina. En Revista de Trabajo $\mathrm{N}^{\circ}$ 7. Año 5. Buenos Aires, Ministerio de Trabajo, Empleo y Seguridad Social de la Nación.

Amargós, O. (2004) "Las transformaciones económicas, los modelos de desarrollo y los desafíos de la educación y formación”. En Jacinto C. (coord.) ¿Educar para que trabajo? Discutiendo rumbos en América Latina. Buenos Aires: Red Etis (IIPE-IDES)/MTCyT y MTEySS/ La Crujía.

Barbetti, P.; D’ Andrea, A.; Sobol, B.; Almirón, M.; Pozzer, J. (2014) “Políticas públicas de formación para el trabajo destinadas a jóvenes provenientes de sectores vulnerables de Chaco y Corrientes. Estudio de tres casos”. En Kairos Revista de Temas Sociales. Año 18 N $^{\circ} 34$. Universidad Nacional de San Luis. Noviembre de 2014. ISSN 1514-9331.

Barbetti, P.; D’Andrea, A.; Sobol, B. (2011) "Características socioeducativas y laborales de los jóvenes de las provincias de Chaco y de Corrientes” En CD Actas del V Congreso de Problemáticas Sociales Contemporáneas. Infancia y Juventudes en los Escenarios Actuales. Organizado por la Universidad Nacional del Litoral. Santa Fe. 14, 15 y 16 de septiembre. EBook ISBN 978-987-657-670-3.

Cortes, R. y Grana, J. (2013) "Empleo no registrado: algunas hipótesis sobre su persistencia 2003-2011”. Ponencia presentada en el XI Congreso de la Asociación Argentina de Estudios del Trabajo (ASET). Buenos Aires: Facultad de Ciencias Económicas (UBA). 
Davalos, P. (2013) "Beneficiarias Asignación Universal por Hijo: características y limitaciones de su participación en el mercado de trabajo”. Revista POIESIS Vol. $7 \mathrm{~N}^{\circ}$ 12, Tubarão, Unisul.

Golovanevsky, L. (2012) "Inserción laboral de los jóvenes en la posconvertibilidad. Unavisión regional”. Ponencia presentada en las II Jornadas Nacionales sobre Estudios Regionales Mercados de Trabajo. Santa Fe: Universidad Nacional del Litoral.

Golovanevsky, L. (2013) “Empleo en la posconvertibilidad.Cambios en la inserción laboral en las regiones argentinas”. Ponencia presentada en el XI Congreso de la Asociación Argentina de Estudios del Trabajo (ASET). Buenos Aires: Facultad de Ciencias Económicas (UBA).

Jacinto, C. (2004) Ante la polarización de oportunidades laborales en América Latina. Un análisis de algunas propuestas recientes en la formación para el trabajo”. En C. Jacinto (coord.), ¿Educar para qué trabajo? Discutiendo rumbos en América Latina. Buenos Aires: Red Etis (IIPE-IDES)/MTCyT y MTEySS/ La Crujía.

Kiss, M. (2005) Corrientes y Neuquén: recursos humanos, competencias y nuevos requerimientos laborales. Demandas de formación técnica y profesional en las provincias de menor desarrollo relativo. Buenos Aires: CEPAL. Disponible en www.cepal.org/publicaciones/ xml/9/20869/dockiss.pdf. Consultado el 8 de septiembre de 2014.

Monteforte, E. y Jaccoud, F. (2013) Las condiciones de vida de los trabajadores y la estructura productiva Argentina. La postconvertibilidad en perspectiva. Ponencia presentada en el XI Congreso de la Asociación Argentina de Estudios del Trabajo (ASET). Buenos Aires, Facultad de Ciencias Económicas (UBA).

OIT Panorama Laboral (2013b). Oficina Regional para América Latina y el Caribe. Lima: OIT.

OIT Trabajo decente y Juventud (2013b). Oficina Regional para América Latina. Lima: OIT.

Panaia, M. y San Martín, M (2004) Introducción General. En Marta Panaia: Crisis Fiscal, mercado de trabajo y nuevas territorialidades en el nordeste argentino. Buenos Aires, La Colmena, pp. 1-4.

Pérez, E. (2007) El desempleo de los jóvenes en Argentina. Seis hipótesis en busca de una explicación. En Revista Estudios del Trabajo No 34. Pag. 79-116. Buenos Aires: Asociación de Especialistas en Estudios del Trabajo (ASET).

Pinazo, G. y Delfini, M. (2014) El empleo informal en la última década. Un análisis com- 
parativo por regiones. Ponencia presentada en las III Jornadas Nacionales sobre Estudios Regionales y Mercados de Trabajo. Jujuy: Universidad Nacional de Jujuy.

Rial, N. (2009) La crisis mundial y la experiencia argentina. En Revista de Trabajo $\mathrm{N}^{\mathrm{o}} 7$. Año 5. Buenos Aires, Ministerio de Trabajo, Empleo y Seguridad Social de la Nación.

Salvia, A. (2013) Juventudes, problemas de empleo y riesgos de exclusión social. El actual escenario de crisis mundial en la Argentina. Berlín: Friedrich-Ebert-Stiftung. En: http:// library.fes.de/pdf-files/iez/09698.pdf [en línea]. Consultado el 22 de noviembre de 2013.

Vera, A. (2009). Los Jóvenes y la Formación para el Trabajo en América Latina. Documento de Trabajo 25. Buenos Aires, CIPPEC (Centro de Implementación de Políticas Públicas para la Equidad y el Crecimiento).

Vezza, E. y Bertranou, F. (2011) “Un nexo por construir: Jóvenes y trabajo decente en Argentina. Radiografía del mercado de trabajo y las principales intervenciones”. BuenosAires: OIT.

Weller, J. (2003) La problemática inserción laboral de los y las jóvenes, Serie Macroeconómica del Desarrollo no ${ }^{\circ}$ 28. Santiago de Chile: CEPAL.

\section{CURRICULUM VITAE}

\section{Pablo Andres Barbetti}

Licenciado en Relaciones Industriales (UNNE, 1997). Magister en Desarrollo Social (2009) Facultad de Humanidades - UNNE. Actualmente realizando el Programa de Doctorado en Humanidades y Ciencias Sociales, de la Universidad Nacional de Misiones.

Docente Investigador Categorizado III del Programa Nacional de Incentivos (2010).

Integrante de equipos de Investigación en el Instituto de Investigaciones Educativas (Facultad de Humanidades - UNNE) y en el Centro de Estudios Sociales (CES - UNNE).

Profesor Adjunto Ordinario a cargo de la Cátedra de "Administración Y Selección de Recursos Humanos”, Carrera de Licenciatura en Ciencias de la Educación. Facultad de Humanidades. Universidad Nacional del Nordeste.

Profesor Adjunto Ordinario a cargo de la Cátedra Seminario de Aplicación Metodológica, Carrera de Relaciones Laborales. Facultad de Ciencias Económicas. Universidad Nacional del Nordeste. Ex - Coordinador de la Carrera Licenciatura en Relaciones Laborales. Facultad de Ciencias Económicas. Universidad Nacional del Nordeste. Sub-director de Departamento de Ciencias de la Educación. Facultad de Humanidades. Universidad Nacional del Nordeste. Miembro de 
la Red SIMEL. Sistema de Información del Mercado Laboral (desde 2003) y miembro de la Red REIJA Red de Investigadores en Juventudes de Argentina (desde 2008). pablobarbetti@hotmail.com

\section{José Antonio Pozzer}

Licenciado en Relaciones Laborales (FCE - UNNE). Doctorando en el Doctorado en Ciencias Sociales de la Universidad Nacional de Entre Ríos. Docente ordinario del Seminario de Aplicación Metodológica (FCE - UNNE). Integrante de comisiones de la FCE - UNNE. Secretario del Departamento de Ciencias Sociales (FCE - UNNE). Becario de Investigación de la Secretaría General de Ciencia y Técnica (UNNE). Integrante de proyectos de investigación acreditados en la línea Políticas Públicas, Juventud, Formación y Trabajo.

japozzer@eco.unne.edu.ar

\section{Blanca Noelia Sobol}

Licenciada en Relaciones Laborales (FCE - UNNE, 2003). Cursando la Especialización en Gestión de las Relaciones del Trabajo (FCE, UNNE). Docente Investigadora Categoría 5 en el Programa de Incentivo a docentes investigadores (Convocatoria 2011, Res. 2013). Profesora Adjunta (interina) en la asignatura Gestión y Administración de Organizaciones Culturales, Lic. en Gestión y Desarrollo Cultural (FADyCC, UNNE). Profesora Adjunta (interina) del Taller de Tesinas, Lic. en RRLL (FCE, UNNE). Docente JTP (ordinaria) con dedicación simple en el Seminario de Aplicación Metodológica. Lic. en RRLL (FCE, UNNE). Docente Auxiliar (ordinaria) con dedicación simple, en la Asignatura Sistemas y Organizaciones de la Lic. en Sistemas de Información, (FaCENA, UNNE). b_sobol@hotmail.com.ar 